\title{
LV-OP-1-5
}

\section{Pure laparoscopic living donor hepatectomy for the right posterior segment graft}

\author{
JaRyung HAN, Young Seok HAN*
}

Division of Hepatobiliay Pancreas Surgery and Liver Transplantation, Department of Surgery, Kyungpook National University Hospital, Daegu, Korea

Introduction: The right posterior segment (RPS) graft was introduced as an alternative to expand donor pool. The first LDLT case using an RPS graft was reported in 2001, the RPS graft has not been widely used in LDLT because of lack of donors with suitable anatomical variation, technical challenge for safe donor hepatectomy and surgical difficulties in recipient operation. We present the 3 cases of pure laparoscopic donor hepatectomy with RPS graft.

Methods: For donor evaluation, we performed laboratory test, abdominal ultrasonography and dynamic computed tomography (CT) scan. Graft selection was determined based on volumetric analysis. In 3 cases of RPS graft, the ratio of the remnant liver volume/ whole liver volume was less than 0.3 and left lobe graft was small for metabolic demand of recipients. All 3 donors had type 3 portal vein variation. The Graft Recipient Weight Ratio (GRWR) was $0.72,0.81$, and 1.34 in each case. Preoperative magnetic resonance cholangiopancreatography (MRCP) was performed to evaluate bile duct anomaly.

Results: In 3 cases, we had no open conversion, intraoperative and postoperative transfusion. Cut surface bile leakage occurred in one case, but bile leakage decreased without procedure or intervention, the donor discharged at postoperative day 9 . He had no more complications. Recipients were recovered uneventfully and discharged.

Conclusions: To meet the donor's safety and recipient's metabolic demand, graft selection is one of the important factors in LDLT, the RPS graft is an alternative to expand donor pool. However, thorough preoperative evaluation to understand the anatomical variation is necessary even for experienced transplant surgeons. 